\title{
Warped Translation Surfaces of Finite Type in Simply Isotropic 3-Spaces
}

\author{
Alev Kelleci Akbay \\ Department of Mathematics, Faculty of Science, Firat University, Elazig, Turkey
}

\section{Article Info}

Keywords: Invariant surface, Laplace operator, Simply isotropic space, Surfaces of finite type

2010 AMS: 53A35, 53A40, 53A55

Received: 26 August 2020

Accepted: 05 November 2020

Available online: 15 December 2020

\begin{abstract}
In this paper, we classify warped translation surfaces being invariant surfaces of i-type, that is, the generating curve has formed by the intersection of the surface with the isotropic $\mathrm{xz}$-plane in the three-dimensional simply isotropic space $\mathbb{I}^{3}$ under the condition

$$
\Delta^{J} x_{i}=\lambda_{i} x_{i}, \quad \text { with } \quad J=I, I I
$$

Here, $\Delta^{J}$ is the Laplace operator with respect to first and second fundamental form and $\lambda_{i}, i=1,2,3$ are some real numbers. Also, as an application, we give some examples for these surfaces and also some explicit graphics of them. All graphics have been plotted with Maple14.
\end{abstract}

\section{Introduction}

Let $\mathbb{E}^{m}$ denotes the $m$-dimensional Euclidean space and $M^{n}$ be a connected $n$-dimensional submanifold in this space. An isometric immersion $\mathbf{x}: M \rightarrow \mathbb{E}^{m}$ is said to be of $k$-type if it can be expressed as a sum of eigenvectors of the Laplace-Beltrami operator of the induced metric $\Delta$, corresponding to $k$ distinct eigenvalues of $\Delta$ :

$$
\mathbf{x}=\mathbf{x}_{0}+\mathbf{x}_{1}+\cdots+\mathbf{x}_{k}, \quad \text { such that } \quad \Delta \mathbf{x}_{i}=\lambda_{i} \mathbf{x}_{i}, \quad i=1, \ldots, k
$$

for a consant vector $\mathbf{x}_{0}$, smooth non-constant functions $\mathbf{x}_{k}$ and $\lambda_{i} \in \mathbb{R}$, [1]. If an isometric immersion $\mathbf{x}$ is of $k$-type, then the submanifold $M$ is said to be of $k$-type [2,3]. In [4], Chen gave a good survey related to finite type submanifolds. In [5], author proved that a submanifold $M^{n}$ in $\mathbb{E}^{m}$ is of 1-type, that is, $\Delta \mathbf{x}=\lambda \mathbf{x}, \lambda \in \mathbb{R}^{+}$, if and only if it is either a minimal submanifold of $\mathbb{E}^{m}(\lambda=0)$ or a minimal submanifold of hypersphere $\mathbb{S}^{m-1}$ in $\mathbb{E}^{m}(\lambda \neq 0)$. In $[6,7]$, by generalizing of this, authors showed that if a hypersurface $M^{n}$ of $\mathbb{E}^{n+1}$ satisfies

$$
\Delta \mathbf{x}=A \mathbf{x}
$$

where $A \in \mathbb{R}^{(n+1) \times(n+1)}$ is a diagonal matrix $A=\operatorname{diag}\left(\lambda_{1}, \ldots, \lambda_{n+1}\right)$. Moreover, Senoussi and Bekkar studied helicoidal surfaces in Euclidean 3-spaces satisfying the condition (1.1), [8]. Furthermore, in [9], Chen gave a detailed paper account of recent development about finite type submanifolds in Euclidean spaces.

On the other hand, very recently, the study on intrinsic (or extrinsic) properties of surfaces in (pseudo-) isotropic spaces has become a research subject for many researchers, see for examples [10]-[17]. Moreover, coordinate finite-type submanifolds 
have been studied in isotropic spaces [18, 19]. Moreover, the study of finite type submanifolds was studied in simply isotropic spaces. In particular, Karacan et. al. studied translation surfaces and surfaces of revolution satisfying

$$
\Delta^{J} x_{i}=\lambda_{i} x_{i}
$$

where $J=I, I I$ and $i=1,2,3$, in these spaces in [20,21] and [22], respectively. Also, in [23], [24] and [25], authors studied affine translation surfaces, helicoidal surfaces and ruled surfaces satisfying the same condition.

In this paper, we are going to study on warped translation surfaces of finite type in three dimensional Isotropic space $\mathbb{I}^{3}$ satisfying (1.2).

\section{Preliminaries}

The simply isotropic 3-space $\mathbb{I}^{3}$ is a Cayley-Klein space defined from the 3-dimensional real projective space $\mathbb{P}^{3}(\mathbb{R})$ with the absolute figure as given $\left\{\omega, d_{1}, d_{2}, f\right\}$. In this space, the homogeneous coordinates $\left[x_{0}: x_{1}: x_{2}: x_{3}\right]$ are presented such that $\omega: x_{0}=0$ is a plane in $\mathbb{P}^{3}(\mathbb{R}), d_{1}: x_{0}=0=x_{1}+\mathrm{i} x_{2}$ and $d_{2}: x_{0}=0=x_{1}-\mathrm{i} x_{2}$ are two complex-conjugate straight lines in the plane, and also $f=[0: 0: 0: 1]$ is a point in the intersection $d_{1} \cap d_{2}$.

The group of motions of $\mathbb{I}^{3}$ is a six-parameter group given by [26]

$$
\begin{aligned}
& \tilde{x}=a_{0}+x \cos \phi-y \sin \phi, \\
& \tilde{y}=a_{1}+x \sin \phi+y \cos \phi, \\
& \tilde{z}=a_{2}+c_{1} x+c_{2} y+z,
\end{aligned}
$$

where $\phi, a_{0}, a_{1}, a_{2}, c_{1}, c_{2} \in \mathbb{R}$. Concerning this group of i-motions, it can be easily seen that these motions are indeed composed of an Euclidean motions onto the $x y$-plane and an affine shear transformation in $z$-direction. Thus, the projection of a point $S(x, y, z)$, in the $z$-direction onto $\mathbb{R}^{2}, \vec{S}(x, y, 0)$ is called the top view of $S$. Let $\vec{A}=\left(x_{1}, x_{2}, x_{3}\right)$ be a vector in $\mathbb{I}^{3}$. If $x_{1}=x_{2}=0$, then $\vec{A}$ is called as isotropic, otherwise non-isotropic. A plane having an isotropic line is said to be an isotropic plane and a line with an isotropic director is an isotropic line.

Given two vectors $\vec{A}=\left(x_{1}, x_{2}, x_{3}\right)$ and $\vec{B}=\left(y_{1}, y_{2}, y_{3}\right)$, the isotropic inner product is calculated by [26]

$$
\langle\vec{A}, \vec{B}\rangle=x_{1} y_{1}+x_{2} y_{2}
$$

Moreover, $M^{2}$ is called as an admissible surface when the metric in $M^{2}$ induced by the isotropic scalar product has rank 2. More precisely, $M^{2}$ parameterized by a $C^{2} \operatorname{map} \mathbf{x}\left(u^{1}, u^{2}\right)=\left(x^{1}\left(u^{1}, u^{2}\right), x^{2}\left(u^{1}, u^{2}\right), x^{3}\left(u^{1}, u^{2}\right)\right)$, is admissible if and only if $X_{12}=x_{1}^{1} x_{2}^{2}-x_{2}^{1} x_{1}^{2} \neq 0$, where $x_{k}^{i}=\partial x^{i} / \partial u^{k}$ and

$$
X_{i j}=\left|x_{1}^{i} x_{2}^{j}-x_{1}^{j} x_{2}^{i}\right|
$$

$[17,26]$. As a result, every admissible $C^{2}$ surface $M^{2}$ can be locally parameterized as $\mathbf{x}\left(u^{1}, u^{2}\right)=\left(u^{1}, u^{2}, f\left(u^{1}, u^{2}\right)\right)$ : one can say that $M$ is in its normal form.

Furthermore, the isotropic first and second fundamental forms I and II, and also their coefficients of the isotropic metric tensor $g_{i j}$ and $h_{i j}$ are given by, respectively, [17]

$$
\begin{aligned}
\mathrm{I} & =g_{i j} u^{i} u^{j} \text { and } g_{i j}=\left\langle\mathbf{x}_{i}, \mathbf{x}_{j}\right\rangle, \\
\mathrm{II} & =h_{i j} u^{i} u^{j} \text { and } h_{i j}=\mathbf{x}_{i j} \cdot \mathbf{N}_{m},
\end{aligned}
$$

where

$$
\mathbf{N}_{m}=\frac{\mathbf{x}_{1} \times \mathbf{x}_{2}}{X_{12}}=\left(\frac{X_{23}}{X_{12}}, \frac{X_{31}}{X_{12}}, 1\right)
$$

Here, we may call $\mathbf{N}_{m}$ the minimal normal because the trace of the Weingarten-like operator $-\mathbf{N}_{m}$ vanishes identically.

Also, the isotropic Gaussian and mean curvatures are given by [17]

$$
K=\frac{h_{11} h_{22}-h_{12}^{2}}{g_{11} g_{22}-g_{12}^{2}} \text { and } H=\frac{1}{2} \frac{g_{11} h_{22}-2 g_{12} h_{12}+g_{22} h_{11}}{g_{11} g_{22}-g_{12}^{2}} .
$$

Note that, a surface in $\mathbb{I}^{3}$ is called as isotropic flat (resp. isotropic minimal ) if $K$ (resp. $H$ ) vanishes. 
Consequently, according to a local coordinate system, the Laplacian $\Delta^{J}, J=I, I I$ in terms of the first and second fundamental forms are defined as usual by, ([20, 21],[26])

$$
\Delta^{I}=-\frac{1}{\sqrt{g_{11} g_{22}-g_{12}^{2}}}\left[\partial_{1}\left(\frac{g_{22} \partial_{1}-g_{12} \partial_{2}}{\sqrt{g}}\right)+\partial_{2}\left(\frac{g_{11} \partial_{2}-g_{12} \partial_{1}}{\sqrt{g}}\right)\right]
$$

and

$$
\Delta^{I I}=-\frac{1}{\sqrt{h_{11} h_{22}-h_{12}^{2}}}\left[\partial_{1}\left(\frac{h_{22} \partial_{1}-h_{12} \partial_{2}}{\sqrt{h_{11} h_{22}-h_{12}^{2}}}\right)+\partial_{2}\left(\frac{h_{11} \partial_{2}-h_{12} \partial_{1}}{\sqrt{h_{11} h_{22}-h_{12}^{2}}}\right)\right]
$$

where $\partial_{i}=\partial / \partial u^{i}$ and $g^{i j}$ is the inverse of the metric, that is, $g^{i k} g_{k j}=\delta_{j}^{i}$. Moreover, throughout paper we will take as $g_{11} g_{22}-g_{12}^{2} \neq 0$ and $h_{11} h_{22}-h_{12}^{2} \neq 0$.

\subsection{Warped translation surface in Simply Isotropic 3-space}

In this work, we will be working on warped translation surfaces being one of the types of invariant surfaces in $\mathbb{I}^{3}$ and some algebraic equations in terms of the Laplacian operator and the coordinate functions of these surfaces. So, in this section, we will work to explain how warped translation surfaces in $\mathbb{I}^{3}$ are parameterized, (For more details, see [17].)

Let $M^{2}$ be a warped translation surface being invariant. So, $M^{2}$ can be parametrized as

$$
M_{\left(a_{0}, a_{1}, 0, c_{1}, c_{2}\right)}^{2}: \mathbf{P}(u, v)=\left(a_{0} v+x(u), a_{1} v, c_{1} v x(u)+z(u)\right),
$$

such that $a_{2}=\left(a_{0} c_{1}+a_{1} c_{2}\right)=0,\left(a_{0}, a_{1}\right),\left(c_{1}, c_{2}\right) \neq(0,0)$. Also, $\phi, a_{0}, a_{1}, a_{2}, c_{1}, c_{2}$ are the real constants as in Eq. (2.1).

Notice that since all simply isotropic invariant surfaces are admissible, throughout paper we will assume that warped translation surfaces are admissible, (see for more details, [17].)

\section{Warped translation surfaces of finite type}

As mentioned in the previous section, the warped translation surfaces can be parametrized as in (2.8) in Isotropic 3-spaces. In this section, we calculate the Laplacian operator $\Delta^{J}$ for these surfaces in $\mathbb{1}^{3}$. And then, we examine the warped translation surfaces satisfying the condition (1.2). Finally, we give the complete classification of these surfaces of finite type in $\mathbb{I}^{3}$.

Now, let us consider on a warped translation surface $M_{\left(a_{0}, a_{1}, 0, c_{1}, c_{2}\right)}^{2}$ defined as in (2.8) with the generating curve $\alpha, \alpha(u)=$ $(u, 0, z(u))$, i.e.,

$$
\mathbf{x}(u, v)=\left(a_{0} v+u, a_{1} v, c_{1} u v+z(u)\right), a_{1}>0 .
$$

Thus, we have

$$
\begin{gathered}
\mathbf{x}_{u}=\left(1,0, c_{1} v+z^{\prime}\right), \\
\mathbf{x}_{v}=\left(a_{0}, a_{1}, c_{1} u\right) .
\end{gathered}
$$

Since $M_{\left(a_{0}, a_{1}, 0, c_{1}, c_{2}\right)}^{2}$ is admissible, i.e., $a_{1} \neq 0$ from (2.2), then $\mathbf{N}_{m}$ the minimal normal defined by (2.4) is computed as

$$
\mathbf{N}_{m}=\left(-c_{1} v-z^{\prime}, \frac{a_{0} z^{\prime}+c_{1}\left(a_{0} v-u\right)}{a_{1}}, 1\right)
$$

By considering (2.3), we obtain the corresponding fundamental forms as [17]

$$
\mathrm{I}=1 \mathrm{~d} u^{2}+2 a_{0} \mathrm{~d} u \mathrm{~d} v+\left(a_{0}^{2}+a_{1}^{2}\right) \mathrm{d} v^{2} \text { and II }=z^{\prime \prime} \mathrm{d} u^{2}+2 c_{1} \mathrm{~d} u \mathrm{~d} v,
$$

and from (2.5), the Gaussian and mean curvatures are

$$
K=-\frac{c_{1}^{2}}{a_{1}^{2}} \text { and } H=-\frac{a_{0} c_{1}}{a_{1}^{2}}+\frac{\left(a_{0}^{2}+a_{1}^{2}\right)}{2 a_{1}^{2}} z^{\prime \prime} .
$$

Finally, the Laplace-Beltrami operators defined as in (2.6) and (2.7) of a warped translation surface are obtained as, respectively,

$$
\Delta^{I}=-\frac{a_{0}^{2}+a_{1}^{2}}{a_{1}^{2}} \frac{\partial^{2}}{\partial u^{2}}+\frac{2 a_{0}}{a_{1}^{2}} \frac{\partial^{2}}{\partial u \partial v}-\frac{1}{a_{1}^{2}} \frac{\partial^{2}}{\partial v^{2}}
$$


and

$$
\Delta^{I I}=-\frac{2}{c_{1}} \frac{\partial^{2}}{\partial u \partial v}+\frac{z^{\prime \prime}}{c_{1}^{2}} \frac{\partial^{2}}{\partial v^{2}}
$$

where $c_{1}$ is a non-zero constant.

Now, firstly we would like to give the following theorem being the classification of parabolic revolution surfaces satisfying (3.2) in $\mathbb{I}^{3}$.

Theorem 3.1. Let $M^{2}$ be a warped translation surface given by (3.1) in $\mathbb{I}^{3}$ such that it satisfies the condition $\Delta^{I} x_{i}=\lambda_{i} x_{i}$, where $\lambda_{i}, i=1,2,3$ are some real constants. Then $M^{2}$ refers to one of the followings:

1. If $\lambda_{1}=\lambda_{2}=0$ and $\lambda_{3}=0$, then the function $z(u)$ is quadratic.

2. If $\lambda_{1}=\lambda_{2}=0$ and $\lambda_{3} \neq 0$, then $\left(a_{0}, a_{1}, 0, c_{1}, c_{2}\right)=\left(a_{0}, a_{1}, 0,0, c_{2}\right)$ and $z$ is given by either

(a) $z(u)=z_{1} \cosh \left(\sqrt{\Lambda_{3}} u\right)+z_{2} \sinh \left(\sqrt{\Lambda_{3}} u\right)$, if $\lambda_{3}>0$, or

(b) $z(u)=z_{1} \cos \left(\sqrt{-\Lambda_{3}} u\right)+z_{2} \sin \left(\sqrt{-\Lambda_{3}} u\right)$, if $\lambda_{3}<0$,

where $\Lambda_{3}=\frac{\lambda_{3} a_{1}^{2}}{a_{0}^{2}+a_{1}^{2}}$.

Proof. Assume that $M^{2}$ is a warped translation surface given by (3.1) and it satisfies the condition (1.2) for $J=I$. Let us take the expressions

$$
\Delta^{I} x=\left(\Delta^{I} x_{1}, \Delta^{I} x_{2}, \Delta^{I} x_{3}\right)
$$

(2.6) and (3.1) together. Thus by a straightforward computation, we get

$$
\Delta^{I} x=\left(0,0,-z^{\prime \prime} \frac{a_{0}^{2}+a_{1}^{2}}{a_{1}^{2}}+\frac{2 a_{0} c_{1}}{a_{1}^{2}}\right) .
$$

So, as $M^{2}$ satisfies the condition $\Delta^{I} x_{i}=\lambda_{i} x_{i}$, where $\lambda_{i}, i=1,2,3$ are some real constants, we have

$$
\begin{aligned}
0 & =\lambda_{1}\left(a_{0} v+u\right), \\
0 & =\lambda_{2} a_{1} v, \\
-z^{\prime \prime} \frac{a_{0}^{2}+a_{1}^{2}}{a_{1}{ }^{2}}+\frac{2 a_{0} c_{1}}{a_{1}{ }^{2}} & =\lambda_{3}\left(c_{1} u v+z\right) .
\end{aligned}
$$

So, from (3.3) and (3.4), we get directly $\lambda_{1}=\lambda_{2}=0$. Now, we will consider on two possibilities coming from (3.5). First, if $\lambda_{3}=0$ then we get the following ODE

$$
\frac{a_{0}^{2}+a_{1}^{2}}{a_{1}^{2}} z^{\prime \prime}-\frac{2 a_{0} c_{1}}{a_{1}^{2}}=0
$$

whose solutions are given as in Case (1) in Theorem 3.1. Secondly, let $\lambda_{3} \neq 0$. By considering $z=z(u)$ in (3.5), we obtain $c_{1}=0$ and

$$
z^{\prime \prime}+\frac{\lambda_{3} a_{1}^{2}}{a_{0}^{2}+a_{1}^{2}} z=0
$$

By taking $\Lambda_{3}=-\frac{\lambda_{3} a_{1}^{2}}{a_{0}^{2}+a_{1}^{2}}$, we can rewrite the last ODE as

$$
z^{\prime \prime}-\Lambda_{3} z=0
$$

whose solutions is given as in Case(2) in Theorem 3.1.

Remark 3.2. By comparing the second equality of (3.2) and (3.6), we conclude that the warped translation surface $M^{2}$ parametrized as in Case (1) in Theorem 3.1 is a isotropic minimal surface in $\mathbb{I}^{3}$.

Remark 3.3. By comparing the first equality of (3.2) and (3.6), we conclude that the warped translation surface $M^{2}$ parametrized as in Case (2) in Theorem 3.1 is a isotropic flat surface in $\mathbb{I}^{3}$.

By considering the above Remark 3.2, we have the following:

Corollary 3.4. A warped translation surface given by (3.1) in the three dimensional simply isotropic space $\mathbb{I}^{3}$ is harmonic if and only if the surface $M^{2}$ is isotropic minimal. 


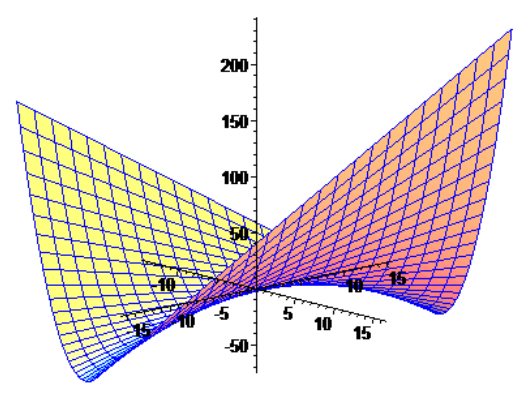

Figure 3.1: An isotropic minimal warped translation surface is parametrized as in Case (1) in Theorem 3.1.

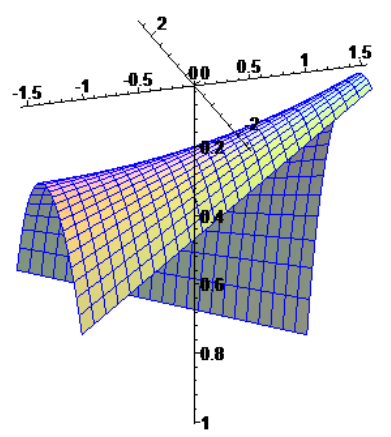

Figure 3.2: An isotropic flat warped translation surface is parametrized as in Case (2a) in Theorem 3.1.

Now, we would like to give some explicit examples of warped translation surfaces satisfing (1.2) for $J=I$ in $\mathbb{I}^{3}$ :

Secondly, we would like to give the following theorem being the classification of warped translation surfaces satisfying (1.2) for $J=I I$ in $\mathbb{I}^{3}$.

Theorem 3.5. Let $M^{2}$ be a warped translation surface given by (3.1) in $\mathbb{I}^{3}$ such that it satisfies the condition $\Delta^{I I} x_{i}=\lambda_{i} x_{i}$, where $\lambda_{i}, i=1,2,3$ are some real constants. Then $M_{\left(a_{0}, a_{1}, 0,0, c_{2}\right)}^{2}$ can be parametrized as

$$
\mathbf{x}(u, v)=\left(a_{0} v+u, a_{1} v, c_{1} u v-\frac{2}{\lambda_{3}}\right), a_{1}>0
$$

Proof. Let $M^{2}$ be a warped translation surface given by (3.1) satisfying (1.2) and

$$
\Delta^{I I} x=\left(\Delta^{I I} x_{1}, \Delta^{I I} x_{2}, \Delta^{I I} x_{3}\right)
$$

By a straightforward computation, we get from (2.7)

$$
\Delta^{I I} x=(0,0,-2)
$$




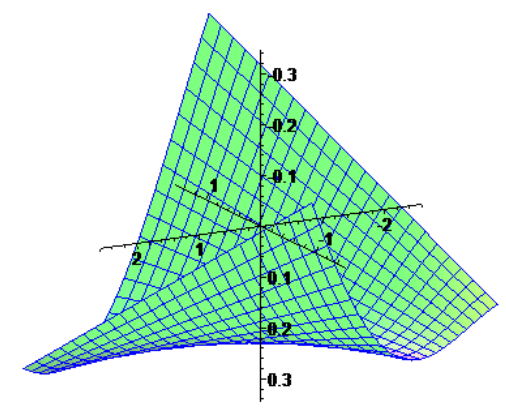

Figure 3.3: An isotropic flat warped translation surface is parametrized as in Case (2b) in Theorem 3.1.

So, as $M^{2}$ satisfies the condition $\Delta^{I I} x_{i}=\lambda_{i} x_{i}$, where $\lambda_{i}, i=1,2,3$ are some real constants, we have

$$
\begin{aligned}
0 & =\lambda_{1}\left(a_{0} v+u\right) \\
0 & =\lambda_{2} a_{1} v \\
-2 & =\lambda_{3}\left(c_{1} u v+z(u)\right) .
\end{aligned}
$$

From (3.7) and (3.8), we get directly, $\lambda_{1}=\lambda_{2}=0$. Now by considering (3.9), we conclude directly that $\lambda_{3} \neq 0$. And so we obtain $c_{1}=0$ and $z=-\frac{2}{\lambda_{3}}$. Thus $M^{2}$ can be parametrized as in Theorem 3.5.

Definition 3.6. A surface in a simply isotropic 3-space, $\mathbb{I}^{3}$ is called as II-harmonic if it satisfies the condition $\Delta^{I I} x=0$, [20].

By considering the above Definition and the proof of Theorem 3.5, we have the following:

Corollary 3.7. There are no II-harmonic warped translation surface in $\mathbb{I}^{3}$.

Now, we would like to give some explicit examples of warped translation surfaces satisfying $\Delta^{I I} x_{i}=\lambda_{i} x_{i}$ in $\mathbb{1}^{3}$ :

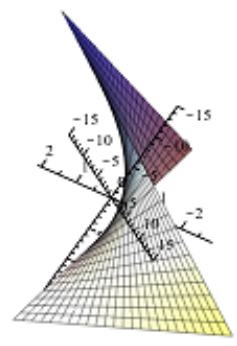

Figure 3.4: A warped translation surface is parametrized as in Theorem 3.5.

\section{Acknowledgments}

The authors would like to thank the referees for their valuable and useful comments that further improve the article.

\section{References}

[1] B. Y. Chen, J. Morvan, T. Nore, Energy, tension and finite type maps, Kodai Math. J., 9 (1986), $406-418$.

[2] B. Y. Chen, Total Mean Curvature and Submanifolds Finite Type, World Scientific, New Jersey, 1984.

[3] O. J. Garay, On a certain class of finite type surfaces of revolution, Kodai Math. J., 11 (1988), $25-31$.

[4] B. Y. Chen, A report on submanifolds of finite type, Soochow J. Math., 22 (1996), 117-337.

[5] T. Tahakashi, Minimal immersions of Riemannian manifolds, J. Math. Soc. Japan, 18 (1966), 380-385. 
[6] F. Dillen, J. Pas, L. Verstraelen, On surfaces of finite type in Euclidean 3-space, Kodai Math. J., 13 (1990), 10-21.

[7] O. J. Garay, An extension of Takahashi's theorem, Geom. Dedicata, 34 (1990), 105-112.

[8] B. Senoussi, M. Bekkar, Helicoidal surfaces with $\Delta^{J} r=$ Ar in 3-dimensional Euclidean space, Stud. Univ. Babes-Bolyai Math., 60 (2015), $437-448$.

[9] B. Y. Chen, Some open problems and conjectures on submanifolds of finite type: recent development, Tamkang J. Math., 45 (2014), 87-108.

[10] M. E. Aydin, A generalization of translation surfaces with constant curvature in the isotropic space, J. of Geo. $107(3)$ (2016), $603-615$.

[11] M. E. Aydin, Constant curvature factorable surfaces in 3-dimensional isotropic space, J. Korean Math. Soc., 55 (1) (2018) $59-7$.

[12] M. E. Aydin, A. Erdur, M. Ergut, Affine factorable surfaces in isotropic spaces, TWMS J. Pure Appl. Math., 11 (2020), 72-88.

[13] M. E. Aydin, I. Mihai, On certain surfaces in the isotropic 4-space, Math. Com., 22(1) (2017), 41-51.

[14] M. E. Aydin, A.O. Ogrenmis, Homothetical and translation hypersurfaces with constant curvature in the isotropic space, BSG proceedings 23, (2016) $1-10$.

[15] A. Kelleci, L. C. B. da Silva, Invariant surfaces with coordinate finite-type Gauss map in simply isotropic space J. Math. Anal. Appl., 495(1) (2021),

[16] L. C. B. da Silva, The geometry of Gauss map and shape operator in simply isotropic and pseudo-isotropic spaces, J. Geom., 110(2) (2019), 31.

[17] L. C. B. da Silva, Differential geometry of invariant surfaces in simply isotropic and pseudo-isotropic spaces, Math. J. Okayama Univ., (in press).

[18] B. Bukcu, M. K. Karacan, D. W. Yoon, Translation surfaces of type-2 in the three-dimensional simply isotropic space $\mathbb{I}_{3}^{1}$, Bull. Korean Math. Soc., 54 (2017), 953-965.

[19] A. Cakmak, M. K. Karacan, S. Kiziltug, Dual surfaces defined by $z=f(u)+g(v)$ in simply isotropic 3-space $\mathbb{I}_{3}^{1}$, Commun. Korean Math. Soc., 34 (2019), 267-277.

[20] B. Bukcu, M. K. Karacan, D. W. Yoon, Translation surfaces in the three-dimensional simply isotropic space $\mathbb{I}_{3}^{1}$ satisfying $\Delta^{I I I} x_{i}=\lambda_{i} x_{i}$, Konuralp J. Math., 4 (2016), 275-281.

[21] M. K. Karacan, D. W. Yoon, B. Bukcu, Translation surfaces in the three-dimensional simply isotropic space $\mathbb{I}_{3}^{1}$, Int. J. Geom. Meth. Mod. Phys., 13 (2016), 1650088 .

[22] M. K. Karacan, D. W. Yoon, B. Bukcu, Surfaces of revolution in the three-dimensional simply isotropic space $\mathbb{I}_{3}^{1}$, Asia Pac. J. Math., 4 (2017), 1-10.

[23] M. E. Aydin, M. Ergut, Affine translation surfaces in the isotropic 3-space, Int. Electron. J. Geom., 10 (2017), 21-30.

[24] M. K. Karacan, D. W. Yoon, S. Kiziltug, Helicoidal surfaces in the three-dimensional simply isotropic space, $\mathbb{I}_{3}^{1}$, Tamkang J. Math., 48 (2017), 123-134

[25] M. K. Karacan, D. W. Yoon, N. Yuksel, Classification of some special types ruled surfaces in simply isotropic 3-space, Analele Universitatii de Vest, Timisoara Seria Matematica - Informatica, 55 (2017), 87-98.

[26] H. Sachs, Isotrope Geometrie des Raumes, Vieweg, Braunschweig/Wiesbaden, 1990. 